\title{
THE ROLE OF INVASIVE AND NON-INVASIVE MEASUREMENTS OF HVPG IN DECISION MAKING IN PATIENTS WITH PORTAL HYPERTENSION AND ESOPHAGEAL VARICES
}

D0l:10.36740/WLek202102128

\author{
Adam Kern' ${ }^{1}$, Tomasz Arłukowicz ${ }^{2}$, Krystian Bojko ${ }^{3}$, Leszek Gromadziński' ${ }^{1}$, Jacek Bil ${ }^{4}$ \\ 'DEPARTMENT OF CARDIOLOGY AND INTERNAL MEDICINE, SCHOOL OF MEDICINE, COLLEGIUM MEDICUM, UNIVERSITY OF WARMIA AND MAZURY \\ IN OLSZTYN, OLSZTYN, POLAND \\ 2DEPARTMENT OF INTERNAL MEDICINE, SCHOOL OF MEDICINE, COLLEGIUM MEDICUM, UNIVERSITY OF WARMIA AND MAZURY IN OLSZTYN, OLSZTYN, POLAND \\ 3DEPARTMENT OF CARDIOLOGY, VOIVODAL SPECIALIST HOSPITAL IN OLSZTYN, OLSZTYN, POLAND \\ ${ }^{4}$ DEPARTMENT OF INVASIVE CARDIOLOGY, CENTRE OF POSTGRADUATE MEDICAL EDUCATION, WARSAW, POLAND
}

\begin{abstract}
Many researchers and clinicians have taken the value of hepatic venous pressure gradient (HVPG) as an essential prognostic factor in subjects with chronic liver disorders. And HVPG alterations characterize a predictive value in subjects at the beginning of the disease (HVPG $6-10 \mathrm{mmHg}$ ) as well as in subjects in whom hemodynamically significant portal hypertension has developed (HVPG $\geq 10 \mathrm{mmHg}$ ).

Our review aims to present the feasibility and applicability of HVPG in modern clinical practice in patients with liver cirrhosis, including invasive and non-invasive methods. HVPG measurement is a feasible method with a favorable safety profile. However, hemodynamically significant portal hypertension also might be determined using non-invasive options as elastography, magnetic resonance imaging, and indices derived from laboratory parameters, e.g., aspartate aminotransferase-to-platelet ratio, platelet count/spleen diameter ratio, or VITRO score. Hepatic vein catheterization with the evaluation of HVPG is the current gold standard for determining portal pressure; however, new non-invasive techniques are nowadays more frequently used.
\end{abstract}

KEY WORDS: elastography, hepatic venous pressure gradient, wedged hepatic vein pressure, spleen stiffness

Wiad Lek. 2021;74(2):321-326

\section{INTRODUCTION}

Many researchers and clinicians have taken the value of hepatic venous pressure gradient (HVPG) as an essential prognostic factor in subjects with chronic liver disorders [1]. HVPG alterations characterize a predictive value in subjects at the beginning of the disease (HVPG $6-10$ $\mathrm{mmHg}$ ) as well as in subjects in whom hemodynamically significant portal hypertension has developed (HVPG $\geq$ $10 \mathrm{mmHg}$ ). In various scenarios, HVPG values are strictly linked to clinical outcomes. The course of esophageal varices, the risk of ascites and encephalopathy as well as the risk of hepatocellular carcinoma development are associated with HVPG in individuals with liver cirrhosis [2-4].

Additionally, subjects responding to pharmacotherapy of portal hypertension (e.g., decrease in HVPG values > $20 \%$ or $\leq 12 \mathrm{mmHg}$ ) characterized a clear risk drop of portal hypertensive complications. They also exhibited a better prognosis [5]. Moreover, early HVPG assessment is a useful marker when used in the course of acute variceal bleeding [6]. Other indications for HVPG measurement are displayed in Table 1.

\section{THE AIM}

Our review aims to present the feasibility and applicability of HVPG in modern clinical practice in patients with liver cirrhosis, including invasive and non-invasive methods.

\section{REVIEW AND DISCUSSION}

\section{HVPG IN COMPUTATIONAL MODELING}

Despite the abovementioned utility of HVPG, we still do not fully acknowledge the influence of various factors involved in the cirrhosis pathogenesis and progression on HVPG values. Therefore, Tang et al. developed a computational model of hepatic circulation [7]. They reaffirmed that the HVPG value is useful in evaluating portal hypertension evoked at the sinusoidal and postsinusoidal level rather than at the presinusoidal level. Nonetheless, several factors impact the HVPG measurement accuracy, e.g., the resistance of presinusoidal portal vessels or the flow in portosystemic collaterals. 


\section{HVPG INVASIVE MEASUREMENT}

Hepatic vein catheterization with HVPG assessment is the current gold standard in verifying portal pressure. HVPG is determined as the difference between the wedged hepatic venous pressure (WHVP) and the free hepatic venous pressure (FHVP). Worth stressing is the fact that WHVP is assessed by occluding the hepatic vein. Blood flow blockage causes pressure equalization with the preceding vascular territory (hepatic sinusoids). This means that WHVP is a surrogate of hepatic sinusoidal pressure, not portal pressure. In healthy subjects, WHVP is only a bit lower (by $\sim 1 \mathrm{mmHg}$ ) than portal pressure; however, in cirrhosis, WHVP gives an exact estimate of portal pressure. It was confirmed both for alcoholic and viral cirrhosis. FHVP corresponds to the not occluded hepatic vein pressure [8].

\section{PROCEDURE DETAILS}

We obtain FHVP by maintaining the catheter's tip free in the hepatic vein at $2-4 \mathrm{~cm}$ from its orifice. FHVP value ought to resemble the value of inferior vena cava pressure. A difference of more than two $\mathrm{mmHg}$ suggests that the catheter is positioned inappropriately or that a hepatic vein obstruction exists.

WHVP is assessed in an occluded hepatic vein. This is obtained by wedging the catheter tip in a small-diameter branch of the hepatic vein or by inflating a balloon at the catheter's tip. Adequate occlusion of the hepatic vein is confirmed by slowly injecting $5 \mathrm{~mL}$ of contrast medium into the vein without observing reflux of the contrast or its washout through communications with other hepatic veins. Occlusion of the hepatic vein by balloon inflation is the preferred technique. The volume of liver circulation sensed by this method is much larger than that attained by wedging the catheter, which reduces measurement variability. Using end-hole, non-balloon catheters is associated with high HVPG variability between various hepatic veins. WHVP should be measured until the value remains stable, preferably for at least 40 seconds. All measurements should be performed at least twice, and all measurements should be recorded. Table 2 presents the elements which should be included in the result. The example procedure is illustrated in Figure 1.

\section{PROCEDURE SAFETY}

HVPG measurement is a procedure with a favorable safety profile. Serious complications are mostly restricted to local injuries at the puncture site (femoral, jugular, or antecubital veins) and include hematoma and bleeding, or rarely arteriovenous fistula or Horner syndrome (in the case of jugular puncture). Ultrasonographic guidance might be additionally used when available, as this tool considerably reduces the risk of procedural complications. The catheter advancement via the right atrium might induce supraventricular arrhythmias (mainly ectopic beats), but in over $90 \%$ of cases, they are self-limited.
Although coagulation disorders are common in patients with cirrhosis, only cases of severe thrombocytopenia (platelet levels $<20 \times 10^{9} / \mathrm{L}$ ) or a low prothrombin ratio (below 30\%) call for the replacement of platelets or transfusion of fresh frozen plasma. The procedure carries only little discomfort. Carried out under moderate conscious sedation $(0.2 \mathrm{mg} / \mathrm{kg}$ intravenous midazolam, which does not influence HVPG measurement), the procedure's acceptability is comparable to that of upper gastrointestinal tract endoscopy [8].

\section{PROCEDURE LIMITATIONS}

Rossle et al. systematically evaluated FVHP measurement accuracy [9]. The study showed that, due to the hepatic vein's conical shape, pressure recordings in the free hepatic vein are substantially affected by the catheter's location. Repeated measurements may be biased by different locations of the catheter's tip and are succumb to manipulation. The authors showed that discrepancies between two locations might exceed the pharmacologic intervention's expected effects ( $10 \%-25 \%$ reduction in the HVPG value), bringing into question the reliability of the procedure using FHVP as an internal reference for HVPG. Therefore, it was recommended to simultaneously measure pressures in the IVC at the level of the hepatic veins' entrance and to use this recording when the difference between the two pressures is above two $\mathrm{mmHg}[8]$.

Also, Silva-Junior et al. focused on the accuracy of the technique. Authors proved that WHVP/FHVP values were more adequate in prognosis determination than WHVP/ inferior vena cava pressure values [10].

Maruyama et al. analyzed the occurrence and hemodynamic features of high-risk esophageal varices with low HVPG values [11]. In the studied population, authors identified $16.4 \%$ of subjects with high-risk esophageal varices and HVPG values below $10 \mathrm{mmHg}$. The venous-venous communication (VVC) incidence was higher in subjects with HVPG values below $10 \mathrm{mmHg}(\mathrm{p}<0.01)$. Subjects with a red sign characterized lower HVPG values (13.3 \pm $4.5 \mathrm{mmHg}$ ) but advanced left gastric vein hemodynamics (velocity $13.2 \pm 3.8 \mathrm{~cm} / \mathrm{s}$; flow volume $217.5 \pm 126.6 \mathrm{~mL} /$ $\mathrm{min})$. On the other hand, subjects without a red sign characterized higher HVPG values $(16.2 \pm 4.6 \mathrm{mmHg}, \mathrm{p}$ $<0.01)$ and worse left gastric vein hemodynamics $(10.9 \pm$ $2.3 \mathrm{~cm} / \mathrm{s}, \mathrm{p}<0.01 ; 160.1 \pm 83.1 \mathrm{~mL} / \mathrm{min}, \mathrm{p}=0.02$ ).

Kim et al. characterized the significance of the classified hemodynamic stage on the basis of HVPG values in subjects with portal hypertension [12]. The following two hemodynamic stage classifications were applied: classification $1(6-9,10-12,13-16,17-20$, and $>20 \mathrm{mmHg})$ and classification $2(6-12,13-20$, and $>20 \mathrm{mmHg})$. Death rates in classification 1 subgroups were $6.3 \%, 6.9 \%$, $18.0 \%, 15.6 \%$, and $34.4 \%$, respectively $(\mathrm{p}<0.01)$. Also, in classification 2 , subgroups mortality rates differed significantly $(\mathrm{p}<0.05)$. Interestingly, in the multivariable model, only classification 2 was a significant prognostic factor in mortality. 


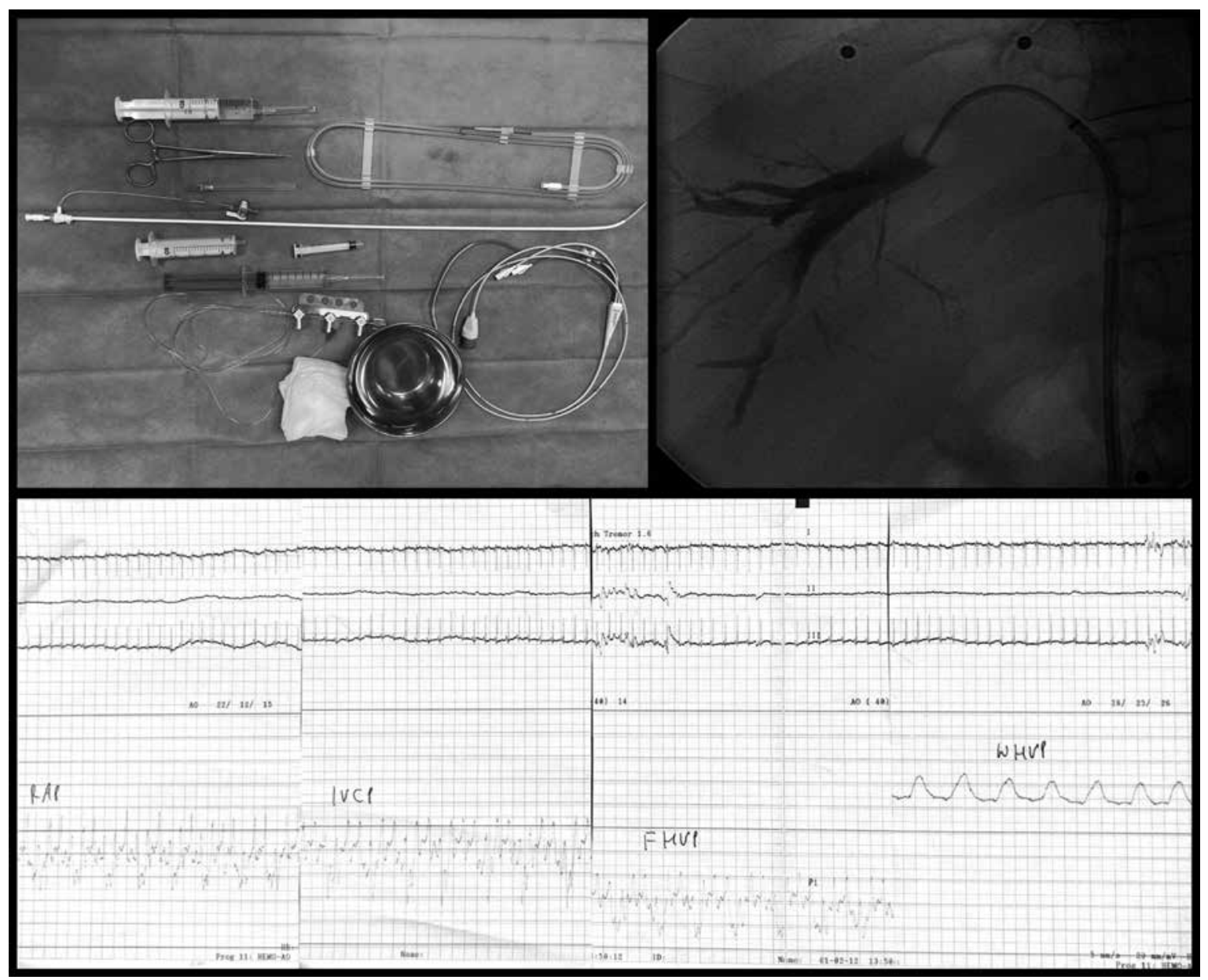

Fig. 1. The example hepatic venous pressure gradient (HVPG) measurement procedure.

\section{ENDOSCOPIC ULTRASOUND-GUIDED PORTAL PRESSURE GRADIENT MEASUREMENT}

Huang et al. characterized the endoscopic ultrasound-guided portal pressure gradient measurement. The procedure was done with the use of a linear echoendoscope, a 25G needle, and a novel compact manometer. Both portal vein and hepatic vein (or inferior vena cava) were reached through a transgastric (or transduodenal) approach. Twenty-eight subjects underwent endoscopic ultrasound-guided portal pressure gradient measurement. Technical success was $100 \%$, and authors registered no complications. HVPG values ranged from $1.5-19 \mathrm{mmHg}$ and highly correlated with clinical features of portal hypertension such as thrombocytopenia $(p=0.04)$, varices presence $(p<0.01)$ or portal hypertensive gastropathy $(\mathrm{p}<0.01)[13]$.

\section{NON-INVASIVE MEASUREMENT TECHNIQUES}

\section{LABORATORY PARAMETERS}

Several non-invasive methods were evaluated as potential screening options for esophageal varices. Some simple parameters were identified as being associated with the presence of varices such as platelet count, prothrombin activity, albumin, alanine aminotransferase, spleen diameter, portal vein diameter, ascites, telangiectasias, or the ChildPugh classification. However, their utility in predicting esophageal varices was not satisfactory.

Aspartate aminotransferase-to-platelet ratio index (APRI) is another option. It was first established in predicting liver fibrosis in subjects with hepatitis C [14]. Mattos et al. disclosed that APRI characterized $64.7 \%$ sensitivity and $43.2 \%$ negative predictive value in predicting esophageal varices in subjects with cirrhosis [15]. More recently, Mandal et al. demonstrated that APRI with a threshold of 0.908 characterized $87.3 \%$ sensitivity, $71.4 \%$ specificity, $92 \%$ positive predictive value, and $60 \%$ negative predictive value [16]. Consequently, APRI might be a helpful tool in indirectly verifying the esophageal varices risk in subjects with liver cirrhosis.

Another proposed index, platelet count/spleen diameter ratio $(\mathrm{PC} / \mathrm{SD})$ with the threshold of 909 , characterized $100 \%$ negative predictive value. The justification for introducing the PC/SD ratio was the idea to correct thrombocytopenia, which is frequently observed in liver diseases. $\mathrm{PC} / \mathrm{SD}$ ratio was also confirmed to be a helpful marker to follow-up patients without varices. Still, validation results were not as astounding as in the original research $(91.5 \%$ sensitivity, $87 \%$ negative predictive value) [17]. This was also verified in more recent studies [18]. 
Table 1. Clinical applications of hepatic venous pressure gradient (HVPG) measurement

Diagnosis of portal hypertension

Classification of portal hypertension (prehepatic, intrahepatic, posthepatic)

Assessment of disease severity and prognosis

Assessment of new therapeutic agents

Response to therapy for portal hypertension

Table 2. The measurement of hepatic venous pressure gradient.

\title{
Key data included in the study protocol
}

1. Access route

2. Type of catheter(s)

3. Hepatic veins used for pressure measurements (right vs middle vs left)

\author{
4. FHVP \\ 5. WHVP \\ 6. HVPG \\ 7. Inferior vena cava pressure \\ 8. Right atrial pressure \\ 9. Complications \\ 10. Additional comments
}

FHVP - free hepatic venous pressure; WHVP - wedged hepatic venous pressure; HVPG - hepatic venous pressure gradient

Hametner et al. evaluated von Willebrand factor antigen (vWF-Ag) to thrombocyte ratio (VITRO score) as a potential marker in predicting hemodynamically significant portal hypertension [19]. Area under the curve (AUC) values showed 0.86 for VITRO score, 0.79 for vWF-Ag, and 0.62 for APRI.

\section{ULTRASOUND}

A doppler ultrasound exam is definitely helpful in the assessment of blood flow in the portal and splanchnic vessels as well as in the imaging of morphological abnormalities co-existing with portal hypertension (e.g., splenomegaly, dilatation of the portal vein system, or development of portosystemic collaterals). The damping index (showing changes in the doppler hepatic vein waveform) corresponds with hemodynamically significant portal hypertension and HVPG values (together with HVPG changes after treatment) [20]. An approach to measuring the resistance of the splenic artery using the splenic doppler pulsatility index together with the portal blood flow is also a reliable option. The obtained results correlated more strongly with HVPG values than any other doppler measurements. Lee et al. proved that the splenic arterial resistive index characterized an improved diagnostic performance in comparison with the liver stiffness evaluated in shear wave elastography [21].

Contrastenhanced ultrasound measurements have recently presented interesting data. The application of subharmonic aided pressure estimation (SHAPE) with the use of perflubutane microbubbles characterized a pretty good correlation with HVPG values. This method was validated in a large multicenter study in the USA (NCT02489045) [21]. Subjects at increased risk for variceal bleeding (HVPG $\geq 12 \mathrm{mmHg}$ ) had a higher mean SHAPE gradient compared to subjects with lower HVPG values $(0.79 \mathrm{~dB} \pm 2.53$ vs. $-4.95 \mathrm{~dB} \pm 3.44 ; \mathrm{p}<0.01)$. The sensitivity was $90 \%$, and the specificity was $80 \%$. The SHAPE gradient between the portal vein and the hepatic vein correlated well with the HVPG values $(r=0.68)$.

\section{ELASTOGRAPHIC METHODS}

Transient elastography was the first method introduced to assess liver stiffness, focused on estimating liver fibrosis. First, transient elastography was applied as an option to invasive liver biopsy for fibrosis staging in subjects with liver disease, especially hepatitis C. Afterwards, transient elastography was proven to characterize a high accuracy in liver cirrhosis recognition. Nowadays, it is generally accepted that transient elastography measurements can also be correlated with the degree of portal pressure. Many researchers tried to overcome liver stiffness limitations on detecting patients with HVPG $>12 \mathrm{mmHg}$, or those with large esophageal varices, by estimating the spleen stiffness. Spleen enlargement is one of the essential diagnostic signs of advanced liver disease. It would be not irrational to hypothesize that, in contrast to liver stiffness, which seems to correlate well with portal pressure only at the initial stages of portal hypertension (HVPG $<10 \mathrm{mmHg}$ ), where the fibrotic component dominates, the spleen stiffness may correlate better with portal hypertension at more advanced stages, when the hyperdynamic circulation and increased portal venous inflow, participates. Studies showed that the combination of liver stiffness and spleen stiffness increased the accuracy of diagnosing patients with advanced compensated cirrhosis not needing screening endoscopy. Karagiannakis et al. disclosed that sequential application of the liver and spleen shear wave elastography predicted hemodynamically significant portal hypertension (HVPG value $>10 \mathrm{mmHg}$ ) with high accuracy [22].

In another study, the following parameters were analyzed: liver stiffness, spleen stiffness, PC/SD, liver stiffness-spleen diameter to platelet ratio score, and variceal risk index [18]. The authors observed significant differences among subjects with or without gastroesophageal varices. The optimal threshold for diagnosing the gastroesophageal varices 
presence was $12.3 \mathrm{kPa}$ in case of liver stiffness and $27 \mathrm{kPa}$ in case of spleen stiffness. However, diagnostic accuracy was moderate (AUC: 0.671 and 0.624 , respectively).

In a meta-analysis, Song et al. assessed the association between spleen stiffness determined in transient elastography and HVPG values. They also evaluated the accuracy of spleen stiffness measurement in recognizing hemodynamically significant portal hypertension [23]. The agreement between spleen stiffness and HVPG values was good, and spleen stiffness characterized good sensitivity and specificity. Unfortunately, the various threshold values among analyzed studies might hamper the significance of obtained results in clinical practice.

In another meta-analysis, Manatsathit et al. analyzed liver stiffness, spleen stiffness, and liver stiffness-spleen size-to-platelet ratio risk score in detecting esophageal varices and high risk/ clinically significant esophageal varices [24]. Spleen stiffness and liver stiffness-spleen size-to-platelet ratio risk score were better than only liver stiffness measurement in detecting esophageal varices. These parameters characterized better sensitivity $(0.90$ and 0.91 vs. 0.85 ), specificity ( 0.73 and 0.76 vs. 0.64$)$, odds ratio (3.24 and 3.35 vs. 2.26 ), and AUC (0.89 and 0.85 vs. 0.82 ). For high risk/clinically significant esophageal varices, spleen stiffness had the highest sensitivity ( 0.87$)$, followed by liver stiffness (0.85) and liver stiffness-spleen size-to-platelet ratio risk score (0.82); however, spleen stiffness had the lowest specificity $(0.52)$, odds ratio (2.09), and AUC (0.81), whereas liver stiffness-spleen size-to-platelet ratio risk score had the highest specificity $(0.77)$, odds ratio (2.74), and AUC (0.86).

And lastly, Piecha et al. disclose that subjects with reducing liver stiffness on the treatment with propranolol characterized a decreased risk for transplantation or death compared to subjects with elevated liver stiffness irrespective of HVPG values [25].

\section{MAGNETIC RESONANCE IMAGING}

Hemodynamic parameters obtained in magnetic resonance imaging correlate with HVPG values. Gouya et al. proved that the azygos flow determined in MRI correlated well with HVPG values (AUC 0.96, 95\% CI $0.91-1.00$ ) in subjects with liver cirrhosis due to hepatitis $C$ or alcohol [26]. In a multicenter study, Palaniyappan et al. showed that a predictive model, including splenic artery velocity, significantly correlated with HVPG values and characterized a good HVPG value predictability in a validation group [27].

\section{COMPUTED TOMOGRAPHY}

Deng et al. performed a metaanalysis, including 17 . They showed that computed tomography sensitivity and specificity in detecting esophageal varices of any size were $87 \%$ and $80 \%$, respectively. For highrisk esophageal varices, the values were $87 \%$ and $88 \%$, respectively [28]. Moreover, in subjects with liver cirrhosis, splenic clearance determined in computed tomography perfusion imaging characterized excellent performance in recognizing the value of $H V P G \geq$ $12 \mathrm{mmHg}$ (94\% sensitivity, 100\% specificity) [29].

\section{CONCLUSIONS}

Over 20 years ago, the measurement of HVPG was applied in managing subjects with liver cirrhosis, mainly in the prevention of the first variceal bleeding. HVPG measurement is a feasible method with a favorable safety profile. However, HVPG measurement is not widely available in clinical practice due to its invasive character and limited feasibility. Additionally, hemodynamically significant portal hypertension may be evaluated using non-invasive methods, such as transient elastography.

\section{REFERENCES}

1. Vorobioff JD, Groszmann RJ. Hepatic venous pressure gradient measurement in pre-primary and primary prophylaxis of variceal hemorrhage. Ann Hepatol. 2013;12(1):22-29.

2. Ripoll C, Groszmann R, Garcia-Tsao G, et al. Hepatic venous pressure gradient predicts clinical decompensation in patients with compensated cirrhosis. Gastroenterology. 2007;133(2):481-488. doi: 10.1053/j. gastro.2007.05.024

3. Feu F, Garcia-Pagan JC, Bosch J, et al. Relation between portal pressure response to pharmacotherapy and risk of recurrent variceal haemorrhage in patients with cirrhosis. Lancet. 1995;346(8982):10561059, doi: 10.1016/s0140-6736(95)91740-3

4. Vorobioff J, Groszmann RJ, Picabea E, et al. Prognostic value of hepatic venous pressure gradient measurements in alcoholic cirrhosis: a 10year prospective study. Gastroenterology. 1996;111(3):701-709. doi: 10.1053/gast.1996.v111.pm8780575

5. Villanueva C, Lopez-Balaguer JM, Aracil C, et al. Maintenance of hemodynamic response to treatment for portal hypertension and influence on complications of cirrhosis. J Hepatol. 2004;40(5):757-765. doi: 10.1016/j.jhep.2004.01.017

6. Monescillo A, Martinez-Lagares F, Ruiz-del-Arbol L, et al. Influence of portal hypertension and its early decompression by TIPS placement on the outcome of variceal bleeding. Hepatology. 2004;40(4):793-801. doi: 10.1002/hep.20386

7. Wang T, Liang F, Zhou Z, et al. A computational model of the hepatic circulation applied to analyze the sensitivity of hepatic venous pressure gradient (HVPG) in liver cirrhosis. J Biomech. 2017;65:23-31. doi: 10.1016/j.jbiomech.2017.09.023

8. Bosch J, Abraldes JG, Berzigotti A, et al. The clinical use of HVPG measurements in chronic liver disease. Nat Rev Gastroenterol Hepatol. 2009;6(10):573-582. doi: 10.1038/nrgastro.2009.149

9. Rossle M, Blanke P, Fritz B, et al. Free Hepatic Vein Pressure Is Not Useful to Calculate the Portal Pressure Gradient in Cirrhosis: A Morphologic and Hemodynamic Study. J Vasc Interv Radiol. 2016;27(8):1130-1137. doi: 10.1016/j.jvir.2016.03.028

10. Silva-Junior G, Baiges A, Turon F, et al. The prognostic value of hepatic venous pressure gradient in patients with cirrhosis is highly dependent on the accuracy of the technique. Hepatology. 2015;62(5):1584-1592, doi: 10.1002/hep.28031

11. Maruyama H, Kobayashi K, Kiyono S, et al. Incidence and hemodynamic feature of risky esophageal varices with lower hepatic venous pressure gradient. Int J Med Sci. 2019;16(12):1614-1620, doi: 10.7150/ ijms.37040

12. Kim TY, Suk KT, Jeong SW, et al. The New Cutoff Value of the Hepatic Venous Pressure Gradient on Predicting Long-Term Survival in Cirrhotic Patients. J Korean Med Sci. 2019;34(33):e223, doi: 10.3346/ jkms.2019.34.e223 
13. Huang JY, Samarasena JB, Tsujino T, et al. EUS-guided portal pressure gradient measurement with a simple novel device: a human pilot study. Gastrointest Endosc. 2017;85(5):996-1001, doi: 10.1016/j. gie.2016.09.026

14. Mattos AZ, Schacher FC, John Neto G, et al. Screening for esophageal varices in cirrhotic patients - Non-invasive methods. Ann Hepatol. 2019;18(5):673-678, doi: 10.1016/j.aohep.2019.06.003

15. Mattos AZ, Alves de Mattos A, Daros LF, et al. Aspartate aminotransferaseto-platelet ratio index (APRI) for the non-invasive prediction of esophageal varices. Ann Hepatol. 2013;12(5):810-814.

16. Mandal AK, Subedi P, Paudel MS, et al. Prediction of Aspartate Aminotransferase to Platelet Ratio Index with Size of Esophageal Varices in Liver Cirrhosis. J Nepal Health Res Counc. 2019;17(1):38-41. doi: 10.33314/jnhrc. 1392

17. Giannini EG, Botta F, Borro P, et al. Application of the platelet count/ spleen diameter ratio to rule out the presence of oesophageal varices in patients with cirrhosis: a validation study based on follow-up. Dig Liver Dis. 2005;37(10):779-785. doi: 10.1016/j.dld.2005.05.007

18. Munoz-Codoceo C, Amo M, Martin A, et al. Diagnostic accuracy of liver and spleen stiffness measured by fibroscan $(R)$ in the prediction of esophageal varices in HCV-related cirrhosis patients treated with oral antivirals. Gastroenterol Hepatol. 2020; doi: 10.1016/j. gastrohep.2020.09.001

19. Hametner S, Ferlitsch A, Ferlitsch M, et al. The VITRO Score (Von Willebrand Factor Antigen/Thrombocyte Ratio) as a New Marker for Clinically Significant Portal Hypertension in Comparison to Other Non-Invasive Parameters of Fibrosis Including ELF Test. PLOS One. 2016;11(2):e0149230. doi: 10.1371/journal.pone.0149230

20. Samarasena JB, Chang KJ. Endoscopic Ultrasound-Guided Interventions for the Measurement and Treatment of Portal Hypertension. Gastrointest Endosc Clin N Am. 2019;29(2):311-320, doi: 10.1016/j.giec.2018.12.004

21. Lee CM, Jeong WK, Lim S, et al. Diagnosis of Clinically Significant Portal Hypertension in Patients with Cirrhosis: Splenic Arterial Resistive Index versus Liver Stiffness Measurement. Ultrasound Med Biol. 2016;42(6):1312-1320, doi: 10.1016/j.ultrasmedbio.2016.01.026

22. Karagiannakis DS, Voulgaris T, Siakavellas SI, et al. Evaluation of portal hypertension in the cirrhotic patient: hepatic vein pressure gradient and beyond. Scand J Gastroenterol. 2018;53(10-11):1153-1164, doi: 10.1080/00365521.2018.1506046

23. Song J, Huang J, Huang $H$, et al. Performance of spleen stiffness measurement in prediction of clinical significant portal hypertension: A meta-analysis. Clin Res Hepatol Gastroenterol. 2018;42(3):216-226, doi: 10.1016/j.clinre.2017.11.002

24. Manatsathit W, Samant H, Kapur S, et al. Accuracy of liver stiffness, spleen stiffness, and LS-spleen diameter to platelet ratio score in detection of esophageal varices: Systemic review and meta-analysis. J Gastroenterol Hepatol. 2018;33(10):1696-1706, doi: 10.1111/jgh.14271
25. Piecha F, Mandorfer M, Peccerella T, et al. Pharmacological decrease of liver stiffness is pressure-related and predicts long-term clinical outcome. Am J Physiol Gastrointest Liver Physiol. 2018;315(4):G484-G494, doi: 10.1152/ajpgi.00392.2017

26. Gouya H, Grabar S, Vignaux 0, et al. Portal hypertension in patients with cirrhosis: indirect assessment of hepatic venous pressure gradient by measuring azygos flow with 2D-cine phase-contrast magnetic resonance imaging. Eur Radiol.2016;26(7):1981-1990, doi: 10.1007/s00330-015-3991-x

27. Palaniyappan N, Cox E, Bradley C, et al. Non-invasive assessment of portal hypertension using quantitative magnetic resonance imaging. J Hepatol. 2016;65(6):1131-1139, doi: 10.1016/j.jhep.2016.07.021

28. Deng H, Qi X, Guo X. Computed tomography for the diagnosis of varices in liver cirrhosis: a systematic review and meta-analysis of observational studies. Postgrad Med. 2017;129(3):318-328, doi: 10.1080/00325481.2017.1241664

29. Talakic E, Schaffellner S, Kniepeiss D, et al. CT perfusion imaging of the liver and the spleen in patients with cirrhosis: Is there a correlation between perfusion and portal venous hypertension? Eur Radiol. 2017;27(10):4173-4180, doi: 10.1007/s00330-017-4788-x

\section{ORCID and contributionship}

Adam Kern - 0000-0003-3341-3701 A, B, D, F

Tomasz Artukowicz - 0000-0002-7066-4211 D-F

Krystian Bojko - 0000-0002-7823-9754 ${ }^{\text {B,D-F }}$

Leszek Gromadziński - 0000-0002-8827-4203 D-F

Jacek Bil - 0000-0002-8724-5611 A,D-F

\section{Conflict of interest}

The Authors declare no conflict of interest.

\section{CORRESPONDING AUTHOR}

Jacek Bil

Department of Invasive Cardiology,

Centre of Postgraduate Medical Education

Woloska 137, 02-507, Warsaw, Poland;

tel.: +48608351353

e-mail: biljacek@gmail.com

Received: 02.01 .2021

Accepted:04.02.2021

A - Work concept and design, B - Data collection and analysis, C - Responsibility for statistical analysis, D-Writing the article, $\mathbf{E}$-Critical review, $\mathbf{F}$ - Final approval of the article 